\title{
Three episodes of non-arteritic posterior ischemic optic neuropathy in the same patient treated with intravenous prostaglandin E1
}

\author{
Robert D Steigerwalt Jr. ${ }^{1, *}$, Antonella Pascarella ${ }^{2}$, Mauro De Angelis ${ }^{2}$, Gabriela Grimaldi ${ }^{3}$, \\ Marcella Nebbioso ${ }^{4}$ \\ ${ }^{1}$ Via A. Brofferio 6, Rome, Italy; \\ ${ }^{2}$ Department of Medical Retina, Ophthalmic Hospital, Rome, Italy; \\ ${ }^{3}$ Via G Belloni 70, Rome, Italy; \\ 4 "La Sapienza", University of Rome, Department of Sense Organs, Rome, Italy.
}

\begin{abstract}
Summary Non-arteritic posterior ischemic optic neuropathy (NA-PION) is a disorder involving reduced blood flow to the retrobulbar portion of the optic nerve. This disorder usually develops acutely, and research has suggested that high-dose steroid therapy soon after the onset of visual loss can result in significant visual improvement. This treatment, however, is not universally successful. The addition of a potent vasodilator could help to restore ocular blood flow. This case report describes the use of prostaglandin E1 (PGE1), a powerful vasodilator of the microcirculation, to treat three separate episodes of NA-PION over five years in the same patient. A 68-year-old white male was first seen in June 2009 with NA-PION in the left eye, and the condition was treated with steroids and PGE1. The patient had a subsequent episode in July 2010 that was treated with steroids and PGE1 and another in May 2014 that was treated with PGE1 alone. Visual acuity improved from 4/10 to 11/10 in 2009, from 4/10 to $11 / 10$ in 2010, and from 5/10 to 10/10 in 2014. No complications due to the use of PGE1 were noted. PGE1 should be considered as a treatment for NA-PION to immediately restore blood flow and potentially improve vision.
\end{abstract}

Keywords: Non-arteritic posterior ischemic optic neuropathy, prostaglandin E1, ophthalmic artery, central retinal artery, color Doppler imaging, hereditary hemochromatosis

\section{Introduction}

Posterior ischemic optic neuropathy (PION) is a disorder involving reduced blood flow to the retrobulbar portion of the optic nerve. PION usually develops acutely and can be classified as surgical, arteritic, or non-arteritic $(1,2)$. The use of high-dose systemic steroids to treat non-arteritic PION (NA-PION) improves visual acuity

(2). Steroid therapy is not universally successful.

Prostaglandin E1 (PGE1), a powerful vasodilator of the microcirculation, improves ocular blood flow in patients with peripheral vascular disease or diabetes (3). Intravenous PGE1 at a dose of $1 \mu \mathrm{g} / \mathrm{kg}$ and steroids

Released online in J-STAGE as advance publication June 15, 2016.

*Address correspondence to:

Dr. Robert D Steigerwalt Jr., Via A. Brofferio 6, 00195 Rome, Italy.

E-mail: r.steigerwalt@libero.it have been used to treat acute non-arteritic anterior ischemic optic neuropathy (NA-AION) and acute arteritic AION (AAION) $(4,5)$. Since NA-PION is a form of ischemia, PGE1 may help.

This case report describes 3 episodes of NA-PION in the same patient over 5 years that were successfully treated with IV PGE1 and steroids. The first episode and treatment in 2009 were previously reported (6). This case report includes blood flow measurements and color Doppler imaging of the ophthalmic artery and the central retinal artery before and after the first treatment. Over 5 years, the same patient had 2 more episodes of NA-PION; one was successfully treated with PGE1 and steroids and the other was treated with PGE1 alone. The results of color Doppler imaging and these 2 subsequent episodes are described here for the first time.

\section{Case Report}

A 68-year-old white male was first seen in June 2009 
eight hours after a loss of visual acuity in his left eye ( 6 ). The right eye was amblyopic and vision was $2 / 10$ in the right eye and 4/10 in the left eye. The patient was being treated for hereditary hemochromatosis. The rest of the ocular examination, including an examination of the optic nerve heads, was normal. A medical examination was performed, initial blood work was done, and an electrocardiogram (EKG) was performed; results revealed no other problems like giant cell arteritis. NAPION in the left eye was preliminarily diagnosed and 50 milligrams (mg) of prednisone was immediately given by mouth. The following morning, $50 \mathrm{mg}$ of prednisone was again given by mouth and color Doppler imaging (CDI) was performed on the retina and optic nerve of both eyes. The blood flow velocity of the central retinal artery as it enters the eye, of the ophthalmic artery as its branches enter the sclera, and of the ophthalmic artery prior to branching was normal in the right eye and markedly reduced in the left eye (Table 1). Blood flow in the nasal and temporal posterior ciliary arteries was markedly reduced in the left eye but normal in the right eye. The patient weighed $70 \mathrm{~kg}$. After a through explanation and written consent, the patient was given $60 \mu \mathrm{g}$ of PGE1 intravenously over $2.5 \mathrm{~h}$. After $1 \mathrm{~h}$, the patient noted a gradual restoration of visual acuity in his left eye. CDI was performed immediately after the first intravenous treatment, revealing a marked increase in blood flow in the vessels of the left eye and a minimal increase in the blood flow in the vessels of the right eye. Blood flow in the left eye was still inferior to that in the right eye (Table 1). The next day, visual acuity remained the same in the right eye at $2 / 10$ and improved to $11 / 10$ in the left eye. The retina and optic nerve head were still normal and the patient was administered $25 \mathrm{mg}$ of prednisone per day for 5 days. Three days after the first infusion of PGE1, CDI of the retrobulbar circulation indicated improvement from the original imaging, but a modest reduction in the flow velocity of the left eye was still present. The patient was intravenously administered $60 \mu \mathrm{g}$ of PGE1. A Humphrey central 30-2 visual field test 1 week posttreatment indicated peripheral scotomas in both eyes. A thorough vascular and medical workup were done immediately after the second infusion. On follow-up visits over the course of a year, visual acuity in the left eye remained 11/10 with no further treatment (6).

In July 2010, 13 months after the first episode, the patient again awoke with decreased visual acuity in his left eye. He was seen 4 hours after onset and he had a Snellen visual acuity of $2 / 10$ in his right amblyopic eye and a visual acuity of $4 / 10$ in his left eye. The rest of the ophthalmic examination was normal, with no swelling of the optic nerve heads. Results of initial medical examinations were normal. The patient was immediately given $50 \mathrm{mg}$ of prednisone by mouth and $60 \mu \mathrm{g}$ of PGE1 intravenously over $2.5 \mathrm{~h}$, and this treatment was repeated the following morning. Twenty-
Table 1. Color Doppler imaging of the ocular vessels before and after PGE1

\begin{tabular}{lll}
\hline Items & Right Eye & Left Eye \\
\hline CRA before PGE1 & $28 / 8 \mathrm{~cm} / \mathrm{sec}$ & $6 / 0 \mathrm{~cm} / \mathrm{sec}$ \\
CRA after PGE1 & $30 / 16 \mathrm{~cm} / \mathrm{sec}$ & $18 / 8 \mathrm{~cm} / \mathrm{sec}$ \\
OA anteriorly before PGE1 & $33 / 12 \mathrm{~cm} / \mathrm{sec}$ & $11 / 2 \mathrm{~cm} / \mathrm{sec}$ \\
OA anteriorly after PGE1 & $36 / 16 \mathrm{~cm} / \mathrm{sec}$ & $19 / 8 \mathrm{~cm} / \mathrm{sec}$ \\
OA retrobulbar before PGE1 & $22 / 10 \mathrm{~cm} / \mathrm{sec}$ & $7 / 0 \mathrm{~cm} / \mathrm{sec}$ \\
OA retrobulbar after PGE1 & $24 / 14 \mathrm{~cm} / \mathrm{sec}$ & $16 / 6 \mathrm{~cm} / \mathrm{sec}$ \\
\hline
\end{tabular}

CRA, central retinal artery; OA - anterior portion, branches of the ophthalmic artery entering the sclera; OA - posterior portion, the ophthalmic artery prior to branching.

five mg of prednisone was administered for 5 days. The patient noted restored vision the afternoon immediately after the first treatment. After the second intravenous administration, vision was $2 / 10$ in the right eye and $11 / 10$ in the left eye. A medical workup revealed no giant cell arteritis. Follow-up ophthalmic visits were normal.

On May 29, 2014, the patient (now 73 years of age) again awoke in the morning with a loss of visual acuity in the left eye. Five hours later, vision was 2/10 in the right eye and 5/10 in the left eye. He had mild cataracts in both eyes. The retina and optic nerve heads were normal. Within 1 hour of the eye examination, 60 $\mu \mathrm{g}$ of PGE1 was administered intravenously without steroids. That afternoon, the patient noticed visual improvement and the following morning $60 \mu \mathrm{g}$ of PGE1 was administered intravenously without steroids. A week later, visual acuity was $2 / 10$ in the right eye and $10 / 10$ in the left eye. The level of vision in the left eye coincided with a mild cataract. A Humphrey central 24-2 visual field test 1 week post-treatment indicated peripheral scotomas in both eyes without central scotomas. Two years later, at the time of this report, the patient's vision has not changed.

\section{Discussion}

PION is a disorder involving reduced blood flow to the retrobulbar portion of the optic nerve $(1,2)$. This condition was noted in the current patient because CDI revealed a reduced blood flow in the ophthalmic artery and central retinal artery of the left eye before treatment with PGE1 in 2009 (Table 1). There was no edema of the optic nerve head, a finding that corroborates the diagnosis of PION. As soon as ischemia was evident, i.e. $24 \mathrm{~h}$ after initial symptoms, intravenous PGE1 was immediately started. PGE1 is a potent vasodilator of the microcirculation (3). CDI immediately after the first administration of PGE1 revealed marked improvement in the ocular and retrobulbar blood flow (Table 1). Along with the increased blood flow, visual acuity also improved immediately after PGE1 in 2009. During the second and third episodes of visual loss over the next 5 years, visual acuity improved immediately after 
treatment with intravenous PGE1. Since NA-PION is ischemia affecting the retrobulbar portion of the optic nerve, the use of a potent vasodilator, PGE1, could be crucial to immediately re-establishing blood flow. In all 3 episodes in the current patient, a through medical workup immediately following treatment was normal except for hereditary hemochromatosis and mild peripheral vascular disease. The optic nerve heads were never swollen and no other signs of giant cell arteritis were noted. Intravenous PGE1 was immediately begun after all 3 episodes without other examinations. Basic blood work and an EKG can be done immediately without delaying treatment. An extensive medical and ocular workup was not done before therapy with PGE1 since a delay in starting treatment could presumably lead to prolonged ischemia and possible permanent damage to the optic nerve. PGE1 has previously been reported to improve visual acuity in cases of NAION and AAION $(4,5)$. In those cases, it was also given shortly after basic blood work and an EKG in order to immediately treat ischemia and avoid permanent damage to the optic nerve.

One intravenous infusion of PGE1 improves peripheral blood flow for up to 4 weeks in patients with peripheral vascular disease (3). Because of this prolonged effect of PGE1, PGE1 was given only two times in each of these 3 epi. Repeated administration could lead to systemic hypotension. To avoid this, $1 \mu \mathrm{g} /$ $\mathrm{kg}$ of PGE1 was only administered intravenously twice after each episode. This patient weighed $70 \mathrm{~kg}$ and was treated with $60 \mu \mathrm{g}$ of PGE1 after each episode.

PGE1 is a safe, potent vasodilator of the peripheral vascular system (the microcirculation or capillary system) that is used to treat patients with signs and symptoms of peripheral vascular disease such as intermittent claudication $(3,7)$. Two days of intravenous administration of PGE1 causes vasodilatation of the capillary system that can last for 4 weeks or longer in patients with peripheral vascular disease. PGE1 is well tolerated with few side effects and can be used in patients who are hypotensive. The systemic blood pressure needs to be monitored frequently (every 15$20 \mathrm{~min}$ ) during intravenous administration $(3,7)$. The main mechanism of action of PGE1 is via vasodilatation of the microcirculation. PGE1 acts directly on the smooth muscle of the vascular wall, leading to vascular dilatation and increased blood flow. This vasodilatation varies depending on the anatomical location and is dose-dependent. PGE1 is also known to inhibit platelet aggregation (8). In addition to the known effects of PGE1 on platelet aggregation, fibrinolysis, blood flow, and viscosity, PGE1 also inhibits monocyte and neutrophil function, suggesting it has anti-inflammatory action as well. Research on gene expression has suggested that several genes in vascular smooth muscle cells and fibroblasts are modified by PGE1 at the transcriptional level. This may contribute to tissue protection in ischemic areas (9). These factors together promote an increase in the capillary flow. PGE1 is rapidly metabolized by oxidation during passage through the pulmonary circulation. It is excreted in the urine as metabolites within about 24 hours (8). This rapid elimination also contributes to its safety.

Steroids, in the form of prednisone, were immediately given to the current patient and were continued orally for a total of 7 days after the first 2 episodes but not after the third. The use of high-dose systemic steroids in a non-randomised retrospective study to treat NA-PION resulted in a significant improvement in visual acuity in comparison to untreated patients (2). Steroids in the same study did not significantly improve visual acuity in patients with arteritic or surgical PION. Another study has suggested the use of systemic corticosteroids in the form of oral prednisone to treat NAION (10). Steroid therapy is, however, not universally accepted. A more recent study using high-dose systemic steroids to treat acute NAION noted no visual or anatomic benefit and several serious complications from steroids (11). However, steroids were used in the current case for another reason, i.e. to try to reduce ischemia-reperfusion (I-R) injury. Ischemia leads to tissue hypoxia, depletion of energy-rich phosphates, accumulation of metabolic waste products including reactive oxygen species, and cellular edema, all of which may cause cellular injury $(12,13)$. The immediate resumption of blood flow is needed to prevent further tissue damage but reperfusion itself may cause further tissue damage and reperfusion injury. Infiltrating leukocytes are thought to play a major role in I-R injury $(12,13)$, and I-R injury was the reason why prednisone was used with PGE1 after the first 2 episodes in the current patient. Steroids were not used after the third episode and their non-use did not appear to have a negative effect. After treatment following the third episode, visual acuity in the left eye was 10/10, which coincides with a cataract in that eye, and the central visual field of the left eye did not appear to be compromised after treatment.

After the first and third episodes, a Humphrey central visual field test 1 week post-treatment indicated peripheral scotomas in both eyes. The visual field of the right eye was worse than that of the left eye, but the fact that the right eye was amblyopic could account for this. A central scotoma was not found in the visual field of the left eye, as is usually noted in NA-PION. However, at the time of the visual field test the patient had already been treated with PGE1, which improved visual acuity to $11 / 10$. Optic disc pallor was not noted on followup visits. This also could be explained by the early treatment.

Since NA-PION is ischemia affecting the retrobulbar portion of the optic nerve, the use of a potent vasodilator could be crucial to immediately re-establishing blood flow. In current case, 3 episodes of NA-PION were promptly treated with PGE1, leading to immediate visual 
improvement without causing any systemic or ocular side effects. Steroids may protect against I-R injury but their use needs to be evaluated further.

\section{References}

1. Sadda SR, Nee M, Miller NR, Biousse V, Newman NJ, Kouzis A. Clinical spectrum of posterior ischemic optic neuropathy. Am J Ophthalmol. 2001; 132:743-750.

2. Hayreh SS. Posterior ischemic optic neuropathy: clinical features, pathogenesis, and management. Eye. 2004; 18:1188-1206.

3. Steigerwalt RD Jr., Belcaro GV, Christopoulos V, Incandela L, Cesarone MR, De Sanctis MT. Ocular and orbital blood flow velocity in patients with peripheral vascular disease and diabetes treated with intravenous prostaglandin E1. J Ocul Pharmacol Ther. 2001; 17:529535.

4. Steigerwalt RD Jr., Cesarone MR, Belcaro G, Pascarella A, De Angelis M, Bacci S. Nonarteritic anterior ischemic optic neuropathy treated with intravenous prostaglandin E1 and steroids. Int J Angiol. 2008; 17:193-196.

5. Steigerwalt RD Jr., Cesarone MR, Belcaro G, Pascarella A, De Angelis M, Gattegna R, Nebbioso M. Arteritic anterior ischemic optic neuropathy treated with intravenous prostaglandin E1 and steroids. Int J Angiol. 2010; 19:113115.

6. Steigerwalt RD Jr., Cesarone MR, Belcaro G, De Angelis M, Pascarella A, Nebbioso M. Non-arteritic posterior ischemic optic neuropathy treated with intravenous prostaglandin E1 and oral corticosteroids. Neuro-
Ophthalmology. 2011; 35:81-84.

7. Cesarone MR, Belcaro G, Nicolaides AN, Griffin M, Geroulakos G, Ramaswami G, Cazaubon M, Barsotti A, Vasdekis S, Christopoulos D, Agus G, Bavera P, Mondani P, Ippolito E, Flenda F. Treatment of severe intermittent claudication: ORACLE-PGE1 short term study. A randomised 40-week study. Evaluation of efficacy and costs. Minerva Cardioangiol. 2002; 50:683-690.

8. Parfitt K. Prostaglandins. In: Martingale. The Complete Drug Reference (Parfitt K, ed.). Pharmaceutical Press, London, UK, 1999; pp. 1411-1413.

9. Schror K, Hohlfeld T. Mechanisms of anti-ischemic action of prostaglandin E1 in peripheral arterial occlusive disease. Vasa. 2004; 33:119-124.

10. Hayreh SS, Zimmerman MB. Non-arteritic anterior ischemic optic neuropathy: role of systemic corticosteroid therapy. Graefes Arch Clin Exp Ophthalmol. 2008; 246:1029-1046.

11. Rebolieda G, Perez-Lòpez M, Casas-LLera P, Contreras I, Munoz-Negrete FJ. Visual and anatomical outcomes of non-arteritic anterior ischemic optic neuropathy with high-dose systemic corticosteroids. Graefes Arch Clin Exp Ophthalmol. 2013; 1:255.

12. Collard CD, Gelman S. Pathophysiology, clinical manifestations and prevention of ischemia-reperfusion injury. Anesthesiology. 2001; 94:1133-1138.

13. Kurokawa T, Takagi H. Mechanisms and prevention of ischemia-reperfusion injury. Transplant Proc. 1999; 31:1775-1776.

(Received May 2, 2016; Revised May 25, 2016; Accepted May 27, 2016) 